\title{
On the existence and uniqueness of solution to Volterra equation on a time scale
}

\author{
Bartłomiej Kluczyński
}

\begin{abstract}
Using a global inversion theorem we investigate properties of the following operator

$$
\begin{gathered}
V(x)(\cdot):=x^{\Delta}(\cdot)+\int_{0}^{\cdot} \nu(\cdot, \tau, x(\tau)) \Delta \tau, \\
x(0)=0
\end{gathered}
$$

in a time scale setting. Under some assumptions on the nonlinear term $v$ we then show that there exists exactly one solution $x_{y} \in W_{\Delta, 0}^{1, p}\left([0,1]_{\mathbb{T}}, \mathbb{R}^{N}\right)$ to the associated integral equation

$$
\left\{\begin{array}{l}
x^{\Delta}(t)+\int_{0}^{t} \nu(t, \tau, x(\tau)) \Delta \tau=y(t) \text { for } \Delta \text {-a.e. } t \in[0,1]_{\mathbb{T}}, \\
x(0)=0
\end{array}\right.
$$

which is considered on a suitable Sobolev space.
\end{abstract}

\section{Introduction}

In this paper using a global invertibility theorem from [18, Theorem 4.G], we consider the following integral operator

$$
V(x)(t):=x^{\Delta}(t)+\int_{0}^{t} \nu(t, \tau, x(\tau)) \Delta \tau,
$$

Key Words: Time scale, Diffeomorphism, Volterra, Operator, Proper operator, Sobolev's space.

2010 Mathematics Subject Classification: 57R50, 26E70, 45D05, 34A12, 46E39.

Received: 18.12.2018

Accepted: 25.02.2019 


$$
x(0)=0,
$$

investigated in the Sobolev space based on a bounded time scale $\mathbb{T}$, such that $0 \in \mathbb{T}$. We obtain, under assumptions given below, that $V$ defines a global diffeomorphism between $W_{\Delta, 0}^{1, p}\left([0,1]_{\mathbb{T}}, \mathbb{R}^{N}\right)$ and $L^{p}\left([0,1]_{\mathbb{T}}, \mathbb{R}^{N}\right)$ for some fixed $p \geq 2$. Spaces $W_{\Delta, 0}^{1, p}\left([0,1]_{\mathbb{T}}, \mathbb{R}^{N}\right)$ and $L^{p}\left([0,1]_{\mathbb{T}}, \mathbb{R}^{N}\right)$ are counterparts of Sobolev and Lebesgue spaces in the continuous setting and ${ }^{\Delta}$ denotes the $\Delta$ derivative while the intergration is with respect to a suitable measure on $\mathbb{T}$ which originates from [16].

As a result of our investigations we will show that for every $y \in L^{p}\left([0,1]_{\mathbb{T}}, \mathbb{R}^{N}\right)$ there exists exactly one solution $x_{y} \in W_{\Delta, 0}^{1, p}\left([0,1]_{\mathbb{T}}, \mathbb{R}^{N}\right)$ to a problem

$$
\left\{\begin{array}{l}
x^{\Delta}(t)+\int_{0}^{t} \nu(t, \tau, x(\tau)) \Delta \tau=y(t) \text { for } \Delta \text {-a.e. } t \in[0,1]_{\mathbb{T}}, \\
x(0)=0 .
\end{array}\right.
$$

The background ideas of solving this problem were inspired by global diffeomorphism theorem from [13] and [8] however in this submission a method from the sources mentioned is modified so that to make it somewhat simpler while retaining the same assertion with same results. Thus it is apparent that the present note is also new as far as equations in the classical setting are concerned. This simplification relies in that we do not use the global inversion tool from [13], so that we do not need to check that the Palais-Smale condition is satisfied. Instead we check that the linear part is proper, the nonlinear compact and the operator is norm-coercive as required by [18, p. 175].

Since we consider integral equations in the time scale setting with techniques which are modification of the approaches known in the classical case, we require some introductory notions from the time scale theory which was introduced by Stefan Hilger in his PhD thesis [12]. The name of this subject and introduction to time scales theory is given in [4]. Many methods of mathematical analysis were reformulated in the time scale setting as seen in $[5]$.

There have been already some literature on the topic of integral equations on time scales. For example in [6] techniques from the continuous case, like Gronwall inequality, continuity and estimate of solutions. Similarly, in [15] we can qualitative properties of solutions for partial integral equations. These include a priori estimates and some convergence type results given with the assumption that a given problem has a solution. Related investigations for Fredholm typ integro-differential equation are contained in [14]. Book [9] is devoted to a classical study of integral equations on a time scale which mimics the classical results in a continuous setting. 
Moreover, there are a few related papers concerning the Volterra type equations. In [3] a special type of discrete version of (1) is considered for which stability results and the existence of bounded solutions are investigated. In [10] the global inversion of non-smooth mappings is considered with possible applications for some other sets of assumptions for equation (1). Some stochastic type equations are to be found in [11].

\section{The assumption and example}

Let $P=[0,1]_{\mathbb{T}} \times \mathbb{R}^{N}$. We assume that function $\nu:\left([0,1]_{\mathbb{T}}\right)^{2} \times \mathbb{R}^{N} \rightarrow \mathbb{R}^{N}$ satisfies

(B1) the function $\nu(\cdot, \tau, \cdot)$ is continuous on $P$ for $\Delta$-a.e. $\tau \in[0,1]_{\mathbb{T}}$,

(B2) the function $\nu_{x}(\cdot, \tau, \cdot)$ is continuous on $P$ for $\Delta$-a.e. $\tau \in[0,1]_{\mathbb{T}}$,

(B3) the function $\nu(t, \cdot, x)$ is measurable on $[0,1]_{\mathbb{T}}$ for all $(t, x) \in P$ and there exist a non-negative functions $c_{1}, d_{1} \in L_{\Delta}^{p}\left(\left([0,1]_{\mathbb{T}}\right)^{2}, \mathbb{R}_{+}^{N}\right)$ such that

$$
|\nu(t, \tau, x)| \leq c_{1}(t, \tau)|x|+d_{1}(t, \tau)
$$

for all $t \in[0,1]_{\mathbb{T}}, \Delta$-a.e. for $\tau \in[0,1]_{\mathbb{T}}$, and for all $x \in \mathbb{R}^{N}$ and function $c_{1}$ satisfies inequality

$$
\int_{0}^{t} c_{1}^{q}(t, \tau) \Delta \tau \leq H_{1} \Delta \text {-a.e. on }[0,1]_{\mathbb{T}}
$$

where $H_{1}$ is some fixed constant,

(B4) the function $\nu_{x}(t, \cdot, x)$ is measurable on $[0,1]_{\mathbb{T}}$ for all $(t, x) \in P$ and there exist a non-negative function $c_{2} \in L_{\Delta}^{p}\left(\left([0,1]_{\mathbb{T}}\right)^{2}, \mathbb{R}_{+}^{N}\right)$ such that

$$
\left|\nu_{x}(t, \tau, x)\right| \leq c_{2}(t, \tau)|x|
$$

for all $t \in[0,1]_{\mathbb{T}}, \Delta$-a.e. for $\tau \in[0,1]_{\mathbb{T}}$, and for all $x \in \mathbb{R}^{N}$ and function $c_{2}$ satisfying inequality

$$
\int_{0}^{t} c_{2}^{q}(t, \tau) \Delta \tau \leq H_{2} \Delta \text {-a.e. on }[0,1]_{\mathbb{T}}
$$

where $H_{2}$ is some fixed constant. 
Here is the example of a nonlinear term satisfying our assumptions.

Let $p=4$, and so $q=\frac{4}{3}$. We put $\mathbb{T}:=\left[0, \frac{1}{2}\right] \cup\left\{1-\frac{1}{n+2}: n \in \mathbb{N}\right\} \cup\{1\}$.

Let $\nu:[0,1]_{\mathbb{T}}^{2} \times \mathbb{R} \rightarrow \mathbb{R}$ be given by

$$
\nu(t, \tau, x):=(t+\tau)^{\frac{3}{2}} \tau^{3} \ln \left(1+x^{2}\right)+t^{\frac{5}{4}}(t-\tau)^{2},
$$

and of course

$$
\nu_{x}(t, \tau, x)=(t+\tau)^{\frac{3}{2}} \tau^{3} \frac{2 x}{1+x^{2}}
$$

We see that

$$
\begin{gathered}
\nu(t, \tau, x) \leq(t+\tau)^{\frac{3}{2}} \tau^{3}|x|+t^{\frac{5}{4}}(t-\tau)^{2} \\
\nu_{x}(t, \tau, x) \leq 2(t+\tau)^{\frac{3}{2}} \tau^{3}|x|
\end{gathered}
$$

Let us put

$$
\begin{gathered}
c_{1}(t, \tau):=(t+\tau)^{\frac{3}{2}} \tau^{3}, \\
d_{1}(t, \tau):=t^{\frac{5}{4}}(t-\tau)^{2}, \\
c_{2}(t, \tau):=2(t+\tau)^{\frac{3}{2}} \tau^{3} .
\end{gathered}
$$

The above functions as polynomials are integrable with the 4 -th power. Consequently we have

$$
\begin{aligned}
\left\|c_{1}\right\|_{L_{\Delta}^{4}}^{4} & =\int_{0}^{1} \int_{0}^{1}|t+\tau|^{6}|\tau|^{12} \Delta \tau \Delta t \leq \int_{0}^{1} \int_{0}^{1}|t+\tau|^{6}|\tau|^{12} d \tau d t \leq 2, \\
\left\|d_{1}\right\|_{L_{\Delta}^{4}}^{4} & =\int_{0}^{1} \int_{0}^{1}|t|^{5}|t-\tau|^{8} \Delta \tau \Delta t \leq\left.\int_{0}^{1} \int_{0}^{1}|t|^{5}|| t|+| \tau\right|^{8} d \tau d t \leq 6, \\
\left\|c_{2}\right\|_{L_{\Delta}^{4}}^{4} & =\int_{0}^{1} \int_{0}^{1} 16|t+\tau|^{6}|\tau|^{12} \Delta \tau \Delta t \leq \int_{0}^{1} \int_{0}^{1} 16|t+\tau|^{6}|\tau|^{12} d \tau d t \leq 32 .
\end{aligned}
$$

Since $t \leq 1$ we see that

$$
\begin{aligned}
& \int_{0}^{1} c_{1}^{\frac{4}{3}}(t, \tau) \Delta \tau=\int_{0}^{1}(t+\tau)^{2} \tau^{4} \Delta \tau \leq \int_{0}^{1}(t+\tau)^{2} \tau^{4} d \tau=\frac{21 t^{2}+35 t+15}{105}<1 \\
& \int_{0}^{1} c_{2}^{\frac{4}{3}}(t, \tau) \Delta \tau=\int_{0}^{1} 2 \sqrt[3]{2}(t+\tau)^{2} \tau^{4} \Delta \tau \leq \int_{0}^{1} 2 \sqrt[3]{2}(t+\tau)^{2} \tau^{4} d \tau \leq 2 \sqrt[3]{2}
\end{aligned}
$$

Therefore, the mapping $V$ given by

$$
V(x)(t)=x^{\Delta}(t)+\int_{0}^{t}(t+\tau)^{\frac{3}{2}} \tau^{3} \ln \left(1+x^{2}(\tau)\right)+t^{\frac{5}{4}}(t-\tau)^{2} \Delta \tau
$$

is a diffeomorphism between $W_{\Delta, 0}^{1, p}\left([0,1]_{\mathbb{T}}, \mathbb{R}^{N}\right)$, and $L_{\Delta}^{p}\left([0,1]_{\mathbb{T}}, \mathbb{R}^{N}\right)$. 


\section{$3 \quad$ Background results}

To simplify a notation, let $a:=\inf \mathbb{T}$, and $b:=\sup \mathbb{T}$ and since $\mathbb{T}$ is bounded, both belong to it.

Define forward jump operator $\sigma: \mathbb{T} \rightarrow \mathbb{T}$ by

$$
\sigma(t):= \begin{cases}\inf \{s \in \mathbb{T}: s>t\} & \text { for } t<b, \\ b & \text { for } t=b,\end{cases}
$$

and backward jump operator $\rho: \mathbb{T} \rightarrow \mathbb{T}$ by

$$
\rho(t):= \begin{cases}\sup \{s \in \mathbb{T}: s<t\} & \text { for } t>a, \\ a & \text { for } t=a .\end{cases}
$$

A point $t \in \mathbb{T}$ is said to be left-dense, left-scattered, right-dense, right-scattered if $\rho(t)=t, \rho(t)<t, \sigma(t)=t, \sigma(t)>t$, respectively. For $f: \mathbb{T} \rightarrow \mathbb{R}$, we define $f^{\sigma}: \mathbb{T} \rightarrow \mathbb{R}$ and the graininess function $\mu: \mathbb{T} \rightarrow[0, \infty)$ by

$$
f^{\sigma}(t):=f(\sigma(t)) .
$$

Define the graininess function $\mu: \mathbb{T} \rightarrow[0, \infty)$ by

$$
\mu(t):=\sigma(t)-t
$$

Set $\mathbb{T}^{\kappa}$ is given by

$$
\mathbb{T}^{\kappa}= \begin{cases}\mathbb{T} & \text { if } b \text { is left-dense, } \\ \mathbb{T} \backslash\{b\} & \text { if } b \text { is left-scattered }\end{cases}
$$

Let $f: \mathbb{T} \rightarrow \mathbb{R}$ and let $t \in \mathbb{T}^{\kappa}$. We define $f^{\Delta}(t)$ to be the number (provided it exists) with the property the given any $\varepsilon>0$, there is a neighborhood $U$ of $t$ (i.e., $U=(t-\delta, t+\delta) \cap \mathbb{T}$ for some $\delta>0)$ such that

$$
\left|f(\sigma(t))-f(s)-f^{\Delta}(t)(\sigma(t)-s)\right|<\varepsilon|\sigma(t)-s| \text { for all } s \in U .
$$

We call $f^{\Delta}(t)$ the delta (or Hilger) derivative of $f$ at $t$. We say that $f$ is the delta (or Hilger) differentiable on $\mathbb{T}^{\kappa}$ provided $f^{\Delta}(t)$ exist for all $t \in \mathbb{T}^{\kappa}$. Then $f: \mathbb{T}^{\kappa} \rightarrow \mathbb{R}$ is called the (delta) derivative of $f$ on $\mathbb{T}^{\kappa}$.

$f:[a, b]_{\mathbb{T}} \rightarrow \mathbb{R}^{N}$ is called rd-continuous provided it is continuous at every right-dense point in $[a, b]_{\mathbb{T}}$ and its left-sided limit exists and it is finite at every left-dense point in $[a, b]_{\mathbb{T}}$.

Space of rd-continuous functions we denote by $C_{r d}\left([a, b]_{\mathbb{T}}, \mathbb{R}^{N}\right)$. 
We say that $p: \mathbb{T} \rightarrow \mathbb{R}$ is regressive if and only if

$$
1+p(t) \mu(t) \neq 0 \text { for all } t \in \mathbb{T}^{\kappa} \text {. }
$$

For $p$ is regressive and rd-continuous, we define the exponential function by

$$
e_{p}(t, a):=\int_{a}^{t} \xi_{\mu(\tau)}(p(\tau)) \Delta \tau \text { for } s, t \in \mathbb{T}
$$

where

$$
\xi_{\mu(\tau)}(p(\tau)):= \begin{cases}p(\tau) & \text { for } t \in \mathbb{T}, \mu(\tau)=0 \\ \frac{\log (1+\mu(\tau) p(\tau))}{\mu(\tau)} & \text { for } t \in \mathbb{T}, \mu(\tau)>0\end{cases}
$$

Remark 3.1 (Theorem 2.48. [4]). Let function $p: \mathbb{T} \rightarrow \mathbb{R}$ will be regressive, $r d$-continuous and $t_{0} \in \mathbb{T}$. If $p$ satisfies the following

$$
1+p(t) \mu(t)>0 \text { for all } t \in \mathbb{T},
$$

then $e_{p}\left(t, t_{0}\right)>0$ for all $t \in \mathbb{T}$.

The ides of integration and measure on $\mathbb{T}$ are taken from [16]. The definitions and theorems given below come from [2] and $[16]$. By $[a, b]_{\mathbb{T}}$ we denote intersection of a interval $[a, b]$ with a time scale $\mathbb{T}$.

Let us define a function $E:[a, b] \rightarrow \mathbb{R}$ by

$$
E(t):=\sup \{s \in \mathbb{T}: s \leq t\}
$$

Suppose that $f: \mathbb{T}^{\kappa} \rightarrow \mathbb{R}^{N}$. Then $f \circ E:[a, b) \rightarrow \mathbb{R}$ is an extension of $f$ to $[a, b)$. Moreover $f$ is constant on "gaps" in $\mathbb{T}$, with value equal to the value of $f$ at the left-hand end of "the gap". We then say that $f$ is measurable, respectively integrable, if $f \circ E$ is measurable, respectively integrable, on the real interval $[a, b]$ in the usual Lebesgue sense. We denote the set of such integrable functions on $\mathbb{T}$ by $L_{\Delta}^{1}\left(\mathbb{T}, \mathbb{R}^{N}\right)$. For any $f \in L_{\Delta}^{1}\left(\mathbb{T}, \mathbb{R}^{N}\right)$ we define the integral of $f$ by

$$
\int_{s}^{t} f \Delta=\int_{s}^{t}(f \circ E)(x) d x
$$

where on the right-hand side the integration is the usual Lebesgue one.

For any $A \subset \mathbb{T}$, let $\chi_{A}$ denote the characteristic function of $A$. We say that $A$ is measurable if $\chi_{A}$ is measurable in the above sense. If $A$ is measurable, we define the integral of $f \in L_{\Delta}^{1}\left(\mathbb{T}, \mathbb{R}^{N}\right)$ over $A$ to be

$$
\int_{A} f \Delta:=\int_{a}^{b} f \chi_{A} \Delta
$$


and we define the measure $\mu_{\mathbb{T}}(A)$ of $A$ to be

$$
\mu_{\mathbb{T}}(A):=\int_{A} 1 \Delta .
$$

Then

$$
\mu_{\mathbb{T}}\left([s, t)_{\mathbb{T}}\right)=t-s \text { for } s, t \in \mathbb{T} .
$$

The only exception to the standard notation is $\{b\}$, for which we have

$$
\mu_{\mathbb{T}}(\{b\})=0,
$$

instead of taking the value of infinity. In our opinion it better reflects that $\mathbb{T}$ unifies both continues and discrete problems.

We define, the space $L_{\Delta}^{p}\left(\mathbb{T}, \mathbb{R}^{N}\right)$ with a norm

$$
\|f\|_{L_{\Delta}^{p}}:=\left(\int_{a}^{b}|f(x)|^{p} \Delta x\right)^{\frac{1}{p}} .
$$

We say that a measurable function $f$ is essentially bounded on $[a, b]_{\mathbb{T}}$ provided there exist a constant $K$ for which $|f(x)| \leq K$ for $\Delta$-a.e. $x \in[a, b]_{\mathbb{T}}$. $L_{\Delta}^{\infty}\left([a, b]_{\mathbb{T}}, \mathbb{R}^{N}\right)$ is equipped with a usual norm

$$
\|f\|_{L_{\Delta}^{\infty}}:=\operatorname{ess} \sup _{x \in[a, b]_{\mathbb{T}}}|f(x)| .
$$

Suppose that $x, y \in \mathbb{R}^{N}$. Then

$$
C_{p}|x-y|^{p} \leq\left(|x|^{p-2} x-|y|^{p-2} y, x-y\right),
$$

where $C_{p}:=\frac{2}{p\left(2^{p-1}-1\right)}$ and symbol $(\cdot, \cdot)$ denotes the inner product in $\mathbb{R}^{N}$.

Lemma 3.2 (Clarkson's inequalities [1]). Let $q$ be conjugate exponent for $p$, i.e. $\frac{1}{p}+\frac{1}{q}=1$ and let $x, y \in \mathbb{R}^{N}$. Then

$$
\left|\frac{x+y}{2}\right|^{p}+\left|\frac{x-y}{2}\right|^{p} \leq \frac{1}{2}\left(|x|^{p}+|y|^{p}\right)
$$

By ([17] p. 33) Hölder's inequality and Minkowski inequality are also valid.

Remark 3.3. Let $1 \leq r<q \leq \infty$. If $x \in L_{\Delta}^{q}\left([a, b]_{\mathbb{T}}, \mathbb{R}^{N}\right)$, then $x \in$ $L_{\Delta}^{r}\left([a, b]_{\mathbb{T}}, \mathbb{R}^{N}\right)$ and from the Hölder inequality we have

$$
\|x\|_{L_{\Delta}^{r}} \leq \mu_{\mathbb{T}}\left([a, b]_{\mathbb{T}}\right)^{\frac{1}{r}-\frac{1}{q}}\|x\|_{L_{\Delta}^{q}}
$$


Therefore

$$
L_{\Delta}^{q}\left([a, b]_{\mathbb{T}}, \mathbb{R}^{N}\right) \hookrightarrow L_{\Delta}^{r}\left([a, b]_{\mathbb{T}}, \mathbb{R}^{N}\right) \text {, i.e. }
$$

the embedding is continuous. In addition, we have an inequality

$$
\|x\|_{L_{\Delta}^{r}}=\left(\int_{a}^{b}|x(t)|^{r} \Delta t\right)^{\frac{1}{r}} \leq\left(\int_{a}^{b}\|x\|_{L_{\Delta}^{\infty}}^{r} \Delta t\right)^{\frac{1}{r}} \leq \mu_{\mathbb{T}}\left([a, b]_{\mathbb{T}}\right)^{\frac{1}{r}}\|x\|_{L_{\Delta}^{\infty}} .
$$

Theorem 3.4. [16] Suppose that $f_{n} \in L_{\Delta}^{p}\left([a, b]_{\mathbb{T}}, \mathbb{R}^{N}\right)$ for $n \in \mathbb{N}$.

a) If $\left\|f_{n}-f\right\|_{L_{\Delta}^{p}} \rightarrow 0$, for some $f \in L_{\Delta}^{p}\left([a, b]_{\mathbb{T}}, \mathbb{R}^{N}\right)$ and $t_{0} \in[a, b]_{\mathbb{T}}$ is rightscattered, then $f_{n}\left(t_{0}\right) \rightarrow f\left(t_{0}\right)$,

b) If $\left\|f_{n}-f\right\|_{L_{\Delta}^{p}} \rightarrow 0$, for some $f \in L_{\Delta}^{p}\left([a, b]_{\mathbb{T}}, \mathbb{R}^{N}\right)$, then there exist increasing sequence $\left(n_{k}\right)_{k \in \mathbb{N}}$ such that $f_{n_{k}} \rightarrow f \Delta$-a.e. on $[a, b]_{\mathbb{T}}$,

c) If $\left(f_{n}\right)_{n \in \mathbb{N}}$ is a Cauchy sequence in $L_{\Delta}^{p}\left([a, b]_{\mathbb{T}}, \mathbb{R}^{N}\right)$, then there exists a unique $f \in L_{\Delta}^{p}\left([a, b]_{\mathbb{T}}, \mathbb{R}^{N}\right)$ such that $\left\|f_{n}-f\right\|_{L_{\Delta}^{p}} \rightarrow 0$.

\section{Sobolev spaces on time scale}

Let $u:[a, b]_{\mathbb{T}} \rightarrow \mathbb{R}^{N}$. We say that $u \in W_{\Delta}^{1, p}\left([a, b]_{\mathbb{T}}, \mathbb{R}^{N}\right)$ if and only if $u \in L_{\Delta}^{p}\left([a, b]_{\mathbb{T}}, \mathbb{R}^{N}\right)$ and if there exists a function $g:\left([a, b]_{\mathbb{T}}\right)^{\kappa} \rightarrow \mathbb{R}^{N}$ such that $g \in L_{\Delta}^{p}\left([a, b]_{\mathbb{T}}, \mathbb{R}^{N}\right)$ and

$$
\int_{a}^{b}\left(u(t), \phi^{\Delta}(t)\right) \Delta t=-\int_{a}^{b}\left(g(t), \phi^{\sigma}(t)\right) \Delta t \text { for all } \phi \in C_{r d, 0}^{1}\left([a, b]_{\mathbb{T}}, \mathbb{R}^{N}\right),
$$

with

$C_{r d, 0}^{1}\left([a, b]_{\mathbb{T}}, \mathbb{R}^{N}\right):=\left\{f:[a, b]_{\mathbb{T}} \rightarrow \mathbb{R}^{N}: f \in C_{r d}^{1}\left([a, b]_{\mathbb{T}}, \mathbb{R}^{N}\right), f(a)=0=f(b)\right\}$

and $C_{r d}^{1}\left([a, b]_{\mathbb{T}}, \mathbb{R}^{N}\right)$ is the set of all continuous functions on $[a, b]_{\mathbb{T}}$ such that they are $\Delta$-differentiable on $[a, b]_{\mathbb{T}}^{\kappa}$ and their $\Delta$-derivatives are $r d$-continuous on $[a, b]_{\mathbb{T}}^{\kappa}$.

Lemma 4.1. [2] Let $f \in L_{\Delta}^{1}\left([a, b]_{\mathbb{T}}, \mathbb{R}^{N}\right)$. Then, a necessary and sufficient condition for the following equality to hold

$$
\int_{a}^{b}\left(f(t), h^{\Delta}(t)\right) \Delta t=0 \text { for every } h \in C_{r d, 0}^{1}\left([a, b]_{\mathbb{T}}, \mathbb{R}^{N}\right),
$$

is the existence of a constant $C \in \mathbb{R}^{N}$, such that $f \equiv C \Delta$-a.e. on $[a, b]_{\mathbb{T}}$. 
We will comment below on the relationship between space $W_{\Delta}^{1, p}\left([a, b]_{\mathbb{T}}, \mathbb{R}^{N}\right)$ and absolutely continuous functions.

Lemma 4.2. [2] Let $f, g:[a, b]_{\mathbb{T}} \rightarrow \mathbb{R}^{N}$ absolutely continuous on $[a, b]_{\mathbb{T}}$. Then their scalar product $(f, g)$ is absolutely continuous on $[a, b]_{\mathbb{T}}$ and the following equality holds

$$
\int_{a}^{b}\left(f^{\Delta} g+f^{\sigma} g^{\Delta}\right)(t) \Delta t=f(b) g(b)-f(a) g(a)=\int_{a}^{b}\left(f g^{\Delta}+f^{\Delta} g^{\sigma}\right)(t) \Delta t .
$$

Lemma 4.3. [2] $f:[a, b]_{\mathbb{T}} \rightarrow \mathbb{R}^{N}$ is absolutely continuous on $[a, b]_{\mathbb{T}}$ if and only if $f$ is delta differentiable $\Delta$-a.e. on $\left([a, b]_{\mathbb{T}}\right)^{\kappa}$ and

$$
f(t):=f(a)+\int_{a}^{t} f^{\Delta}(s) \Delta s \text { for all } t \in \mathbb{T} .
$$

Theorem 4.4. Suppose that $u \in W_{\Delta}^{1, p}\left([a, b]_{\mathbb{T}}, \mathbb{R}^{N}\right)$ and that (6) holds for some $g \in L_{\Delta}^{p}\left([a, b]_{\mathbb{T}}, \mathbb{R}^{N}\right)$. Then, there exists a unique absolutely continuous function $x$, such that

$$
\begin{array}{ll}
u=x & \Delta \text {-a.e. on }[a, b]_{\mathbb{T}}, \\
g=x^{\Delta} & \Delta \text {-a.e. on }[a, b]_{\mathbb{T}} .
\end{array}
$$

Proof. Let us define a function $v:[a, b]_{\mathbb{T}} \rightarrow \mathbb{R}^{N}$ as

$$
v(t)=\int_{a}^{t} g(s) \Delta s
$$

Let $h \in C_{r d, 0}^{1}\left([a, b]_{\mathbb{T}}, \mathbb{R}^{N}\right)$. Since $v$ is absolutely continuous and since $(7)$ holds, we see that

$$
\int_{a}^{b}\left(v(t), h^{\Delta}(t)\right) \Delta t=-\int_{a}^{b}\left(v^{\Delta}(t), h^{\sigma}(t)\right) \Delta t
$$

We have $v^{\Delta}=g \Delta$-a.e. on $[a, b]_{\mathbb{T}}$. Since $g$ is the generalized derivative of the function $u$, we have

$$
\int_{a}^{b}\left(u(t), h^{\Delta}(t)\right) \Delta t=-\int_{a}^{b}\left(g(t), h^{\sigma}(t)\right) \Delta t
$$

for every $h \in C_{r d, 0}^{1}\left([a, b]_{\mathbb{T}}, \mathbb{R}^{N}\right)$. Therefore

$$
\int_{a}^{b}\left(v(t)-u(t), h^{\Delta}(t)\right) \Delta t=-\int_{a}^{b}\left(v^{\Delta}(t)-g(t), h^{\sigma}(t)\right) \Delta t=0 .
$$


From Lemma 4.1 there exists a constant $C_{0} \in \mathbb{R}^{N}$ such that $v-u \equiv C_{0} \Delta$ almost everywhere on $[a, b]_{\mathbb{T}}$. As a consequence we conclude that the function $x:[a, b]_{\mathbb{T}} \rightarrow \mathbb{R}^{N}$ defined as $x(t)=v(t)-C_{0}$ for all $t \in[a, b]_{\mathbb{T}}$ is the unique absolutely continuous function satisfying (9).

In this way, we obtain a vector space $W_{\Delta}^{1, p}\left([0,1]_{\mathbb{T}}, \mathbb{R}^{N}\right)$ as a space of absolutely continuous functions with norm defined by the formula

$$
\|x\|_{W_{\Delta}^{1, p}}:=\left(\int_{0}^{1}|x(t)|^{p} \Delta t\right)^{\frac{1}{p}}+\left(\int_{0}^{1}\left|x^{\Delta}(t)\right|^{p} \Delta t\right)^{\frac{1}{p}} .
$$

Let us define a subspace of space $W_{\Delta}^{1, p}\left([0,1]_{\mathbb{T}}, \mathbb{R}^{N}\right)$, denoted by

$$
W_{\Delta, 0}^{1, p}\left([0,1]_{\mathbb{T}}, \mathbb{R}^{N}\right):=\left\{f \in W_{\Delta}^{1, p}\left([0,1]_{\mathbb{T}}, \mathbb{R}^{N}\right): f(0)=0\right\},
$$

with a norm $\|\cdot\|_{W_{\Delta, 0}^{1, p}}$ given by

$$
\|x\|_{W_{\Delta, 0}^{1, p}}:=\left(\int_{0}^{1}\left|x^{\Delta}(t)\right|^{p} \Delta t\right)^{\frac{1}{p}}
$$

Theorem 4.5 (Poincáre Inequality). For any $x \in W_{\Delta, 0}^{1, p}\left([0,1]_{\mathbb{T}}, \mathbb{R}^{N}\right)$ the following inequality holds

$$
\|x\|_{W_{\Delta}^{1, p}} \leq 2\|x\|_{W_{\Delta, 0}^{1, p}}
$$

Proof. Let $x \in W_{\Delta, 0}^{1, p}\left([0,1]_{\mathbb{T}}, \mathbb{R}^{N}\right)$. Since $x(0)=0$

$$
|x(t)|=|x(t)-x(0)|=\left|\int_{0}^{t} x^{\Delta}(s) \Delta s\right| \leq\left\|x^{\Delta}\right\|_{L_{\Delta}^{1}},
$$

for every $t \in[0,1]_{\mathbb{T}}$, we see that $\|x\|_{L_{\Delta}^{\infty}} \leq\left\|x^{\Delta}\right\|_{L_{\Delta}^{1}}$, and applying Remark 3.3 we obtain $\|x\|_{L_{\Delta}^{p}} \leq\left\|x^{\Delta}\right\|_{L_{\Delta}^{p}}$.

Remark 4.6. Observe that by Theorem 4.5 norms $\|\cdot\|_{W_{\Delta, 0}^{1, p}},\|\cdot\|_{W_{\Delta}^{1, p}}$ are equivalent on $W_{\Delta, 0}^{1, p}\left([0,1]_{\mathbb{T}}, \mathbb{R}^{N}\right)$. Indeed, for any $x$

$$
\|x\|_{W_{\Delta, 0}^{1, p}} \leq\|x\|_{W_{\Delta}^{1, p}}=\left\|x^{\Delta}\right\|_{L_{\Delta}^{p}}+\|x\|_{L_{\Delta}^{p}} \leq\left\|x^{\Delta}\right\|_{L_{\Delta}^{p}}+\left\|x^{\Delta}\right\|_{L_{\Delta}^{p}}=2\|x\|_{W_{\Delta, 0}^{1, p}} .
$$

Theorem 4.7. Space $\left(W_{\Delta, 0}^{1, p}\left([0,1]_{\mathbb{T}}, \mathbb{R}^{N}\right),\|\cdot\|\right)$ is reflexive. 
Proof. Let $\left(x_{n}\right)_{n \in \mathbb{N}}$ be Cauchy sequence in $W_{\Delta, 0}^{1, p}\left([0,1]_{\mathbb{T}}, \mathbb{R}^{N}\right)$. Therefore, there exist a sequence $\left(x_{n}^{\Delta g}\right)_{n \in \mathbb{N}}$ of generalized derivatives such that

$\int_{0}^{1}\left(x_{n}(t), \phi^{\Delta}(t)\right) \Delta t=-\int_{0}^{1}\left(x_{n}^{\Delta g}(t), \phi^{\sigma}(t)\right) \Delta t$ for any $\phi \in C_{r d, 0}^{1}\left([a, b]_{\mathbb{T}}, \mathbb{R}^{N}\right)$.

This means that $\left(x_{n}\right)_{n \in \mathbb{N}}$ and $\left(x_{n}^{\Delta g}\right)_{n \in \mathbb{N}}$ are Cauchy sequences in $L_{\Delta}^{p}\left([0,1]_{\mathbb{T}}, \mathbb{R}^{N}\right)$. From Theorem 3.4 we know there exist $x, x^{\Delta} \in L_{\Delta}^{p}\left([0,1]_{\mathbb{T}}, \mathbb{R}^{N}\right)$ such that

$$
\begin{aligned}
& \left\|x_{n}-x\right\|_{L_{\Delta}^{p}} \rightarrow 0, \\
& \left\|x_{n}^{\Delta g}-x^{\Delta g}\right\|_{L_{\Delta}^{p}} \rightarrow 0 .
\end{aligned}
$$

Moreover, applying Hölder inequality we obtain

$$
\begin{aligned}
& \int_{0}^{1}\left(x_{n}(t)-x(t)\right) \phi^{\Delta}(t) \Delta t \leq\left\|x_{n}-x\right\|_{L_{\Delta}^{p}}\left\|\phi^{\Delta}\right\|_{L_{\Delta}^{q}} \rightarrow 0, \\
& \int_{0}^{1}\left(x_{n}^{\Delta g}(t)-x^{\Delta g}(t)\right) \phi^{\sigma}(t) \Delta t \leq\left\|x_{n}^{\Delta g}(t)-x^{\Delta g}(t)\right\|_{L_{\Delta}^{p}}\left\|\phi^{\sigma}\right\|_{L_{\Delta}^{q}} \rightarrow 0 .
\end{aligned}
$$

Consequently

$$
\begin{aligned}
\int_{0}^{1} x(t) \phi^{\Delta}(t) \Delta t & =\lim _{n \rightarrow \infty} \int_{0}^{1} x_{n}(t) \phi^{\Delta}(t) \Delta t \\
& \left.=\lim _{n \rightarrow \infty} \int_{0}^{1} x_{n}^{\Delta g}(t)\right) \phi^{\sigma}(t) \Delta t=\int_{0}^{1} x^{\Delta g}(t) \phi^{\sigma}(t) \Delta t .
\end{aligned}
$$

It remains to show that $x(0)=0$. This follows since $x_{n}$ converges uniformly on $[0,1]_{\mathbb{T}}$. Hence $W_{\Delta, 0}^{1, p}\left([0,1]_{\mathbb{T}}, \mathbb{R}^{N}\right)$ is a complete normed vector space, as a closed subspace of such a space.

Now we investigate the uniform convexity. Let us assume that $x, y \in$ $W_{\Delta, 0}^{1, p}\left([0,1]_{\mathbb{T}}, \mathbb{R}^{N}\right)$ are such that $\|x\|_{W_{\Delta, 0}^{1, p}}=1,\|y\|_{W_{\Delta, 0}^{1, p}}=1,\|x-y\|_{W_{\Delta, 0}^{1, p}} \geq 1$.

From Lemma 3.2 we have

$$
\begin{aligned}
\left\|\frac{x+y}{2}\right\|_{W_{\Delta, 0}^{1, p}}^{p}+\left\|\frac{x-y}{2}\right\|_{W_{\Delta, 0}^{1, p}}^{p} & =\int_{0}^{1}\left|\frac{x^{\Delta}(t)+y^{\Delta}(t)}{2}\right|^{p} \Delta t+\int_{0}^{1}\left|\frac{x^{\Delta}(t)-y^{\Delta}(t)}{2}\right|^{p} \Delta t \\
& \leq \int_{0}^{1} \frac{1}{2}\left(\left|x^{\Delta}(t)\right|^{p}+\left|y^{\Delta}(t)\right|^{p}\right) \Delta t=1 .
\end{aligned}
$$

Therefore

$$
\left\|\frac{x+y}{2}\right\|_{W_{\Delta, 0}^{1, p}} \leq\left(1-\left(\frac{\varepsilon}{2}\right)^{p}\right)^{\frac{1}{p}} \leq 1-\frac{1}{p}\left(\frac{\varepsilon}{2}\right)^{p} .
$$

From Milman-Pettis Theorem we obtain the reflexivity of $W_{\Delta, 0}^{1, p}\left([0,1]_{\mathbb{T}}, \mathbb{R}^{N}\right)$. 
Now we introduce the Bielecki norm. Let $\beta>0, x \in L_{\Delta}^{p}\left([0,1]_{\mathbb{T}}, \mathbb{R}^{N}\right)$

$$
\|x\|_{\beta, L_{\Delta}^{p}}:=\left(\int_{0}^{1} \frac{1}{e_{\beta}^{\sigma}(t, 0)}|x(t)|^{p} \Delta t\right)^{\frac{1}{p}}
$$

where $e_{\beta}^{\sigma}(t, 0)$ is a superposition of a jump operator and the exponent on the time scale defined in (3). Using the monotonicity of the exponent function. We see

$$
\left(\int_{0}^{1}|x(t)|^{p} \Delta t\right)^{\frac{1}{p}} \geq\left(\int_{0}^{1} \frac{1}{e_{\beta}^{\sigma}(t, 0)}|x(t)|^{p} \Delta t\right)^{\frac{1}{p}} \geq\left(\int_{0}^{1} \frac{1}{e_{\beta}^{\sigma}(1,0)}|x(t)|^{p} \Delta t\right)^{\frac{1}{p}} .
$$

Therefore both norms are equivalent, i.e.

$$
\|x\|_{L_{\Delta}^{p}} \geq\|x\|_{\beta, L_{\Delta}^{p}} \geq \frac{1}{e_{\beta}^{\sigma}(1,0)}\|x\|_{L_{\Delta}^{p}} .
$$

Remark 4.8. For every $x \in W_{\Delta, 0}^{1, p}\left([0,1]_{\mathbb{T}}, \mathbb{R}^{N}\right)$ due to integration by parts we obtain

$$
\begin{aligned}
& \text { i) }\|x\|_{\beta, L_{\Delta}^{p}} \leq\left(\frac{1}{\beta}\right)^{\frac{1}{p}}\|x\|_{\beta, W_{\Delta, 0}^{1, p}}, \\
& \text { ii) }\left\|\int_{0}^{\cdot}|x(\tau)| \Delta \tau\right\|_{\beta, L_{\Delta}^{p}} \leq\left(\frac{1}{\beta}\right)^{\frac{2}{p}}\|x\|_{\beta, W_{\Delta, 0}^{1, p}} .
\end{aligned}
$$

\section{Main results}

Let $f: E \rightarrow B$ be mapping between Banach spaces $E$ and $B . \quad f$ is called proper iff the preimage $f^{-1}(C)$ of every compact set $C$ in $B$ is also compact.

Theorem 5.1 (Theorem 4.G [18]). Let $f: E \rightarrow B$ be a local diffeomorphism, at every point of $E$. Then $f$ is a diffeomorphism iff $f$ is proper.

Theorem 5.2 ([18] p. 175). Let $f: E \rightarrow B$ be a continuous coercive norm mapping between the two Banach spaces, i.e. $\|V(x)\| \rightarrow \infty$ as $\|x\| \rightarrow \infty$. Then $f$ is proper if there exist functions $g, h: E \rightarrow B$ such that $g$ is proper, $h$ is compact and $f=g+h$.

Theorem 5.3 (Mean Value Theorem 3.2.6 [7]). Assume $f: E \rightarrow B$ has the directional derivative at all points of the line segment joining points $a, b \in E$ in the direction of this segment i.e., $f_{G}^{\prime}(a+t(b-a))(a-b)$ exist for all $t \in[0,1]$. If the mapping $t \mapsto f_{G}^{\prime}(a+t(b-a))(a-b)$ is continuous on $[0,1]$, then

$$
f(b)-f(a)=\int_{0}^{1} f_{G}^{\prime}(a+t(b-a))(a-b) d t .
$$


Remark 5.4. Let $[s, t)_{\mathbb{T}}$ be an subset of some a time scale $\mathbb{T}$ and let $2 \leq p<$ $\infty$. The functional $\xi$ given by

$$
\xi(u):=\int_{s}^{t}|u(\tau)|^{p} \Delta \tau,
$$

is of class $\mathrm{C}^{1}\left(L_{\Delta}^{p}\left([s, t)_{\mathbb{T}}, \mathbb{R}\right), \mathbb{R}\right)$ and

$$
\left(\xi^{\prime}(u), h\right)=p \int_{s}^{t}|u(\tau)|^{p-2} u(\tau) h(\tau) \Delta \tau .
$$

In all results which follow it is assumed that conditions (B1)-(B4) are satisfied.

Theorem 5.5. Mapping $W_{\Delta, 0}^{1, p}\left([0,1]_{\mathbb{T}}, \mathbb{R}^{N}\right) \ni x \mapsto x^{\Delta} \in L_{\Delta}^{p}\left([0,1]_{\mathbb{T}}, \mathbb{R}^{N}\right)$ is proper.

Proof. Let $V$ be compact in $L_{\Delta}^{p}\left([0,1]_{\mathbb{T}}, \mathbb{R}^{N}\right)$ and $W:=\left\{w \in W_{\Delta, 0}^{1, p}\left([0,1]_{\mathbb{T}}, \mathbb{R}^{N}\right)\right.$ : $\left.w^{\Delta} \in V\right\}$. Consider any sequence $\left(w_{n}\right)_{n \in \mathbb{N}}$ in $W$, and define sequence $v_{n}:=$ $w_{n}^{\Delta}$, for all $n \in \mathbb{N}$. Note that $\left(v_{n}\right)_{n \in \mathbb{N}}$ has a convergent subsequence $\left(v_{n_{k}}\right)_{k \in \mathbb{N}}$ to $v_{0} \in V$ and consequently $\left(v_{n_{k}}\right)_{k \in \mathbb{N}}$ is a Cauchy sequence. By equality

$$
\left\|w_{n_{k}}-w_{n_{j}}\right\|_{W_{\Delta, 0}^{1, p}}=\left\|w_{n_{k}}^{\Delta}-w_{n_{j}}^{\Delta}\right\|_{L_{\Delta}^{p}}=\left\|v_{n_{k}}-v_{n_{j}}\right\|_{L_{\Delta}^{p}}
$$

$\left(w_{n_{k}}\right)_{k \in \mathbb{N}}$ is a Cauchy sequence in $W_{\Delta, 0}^{1, p}\left([0,1]_{\mathbb{T}}, \mathbb{R}^{N}\right)$, thus $\left(w_{n_{k}}\right)_{k \in \mathbb{N}}$ is convergent to some $w_{0}$. Thus from equality

$$
\left\|w_{n_{k}}-w_{0}\right\|_{W_{\Delta, 0}^{1, p}}=\left\|w_{n_{k}}^{\Delta}-w_{0}^{\Delta}\right\|_{L_{\Delta}^{p}}=\left\|v_{n_{k}}-w_{0}^{\Delta}\right\|_{L_{\Delta}^{p}}
$$

we get $w_{0}^{\Delta}=v_{0}$. This shows that $W$ is compact.

Theorem

5.6.

Mapping $W_{\Delta, 0}^{1, p}\left([0,1]_{\mathbb{T}}, \mathbb{R}^{N}\right) \ni x \mapsto \int_{0}^{t} \nu(t, \tau, x(\tau)) \Delta \tau \in L_{\Delta}^{p}\left([0,1]_{\mathbb{T}}, \mathbb{R}^{N}\right)$ is compact.

Proof. Let $\left(x_{n}\right)_{n \in \mathbb{N}}$ by weakly convergent to some $x_{0} \in W_{\Delta, 0}^{1, p}\left([0,1]_{\mathbb{T}}, \mathbb{R}^{N}\right)$. Then $\left(x_{n}\right)_{n \in \mathbb{N}}$ converges to $x_{0}$ in $L_{\Delta}^{p}\left([0,1]_{\mathbb{T}}, \mathbb{R}^{N}\right)$. For functions $c_{1}, d_{1} \in$ $L_{\Delta}^{p}\left([0,1]_{\mathbb{T}}^{2}, \mathbb{R}_{+}^{N}\right)$ from condition (B3) we have

$$
\left|\nu\left(t, \tau, x_{n}(\tau)\right)-\nu\left(t, \tau, x_{0}(\tau)\right) \Delta \tau\right| \leq 2 M c_{1}(t, \tau)+d(t, \tau),
$$

and by the Dominated Convergence Theorem we obtain

$$
\left(\int_{0}^{1}\left(\int_{0}^{t} \nu\left(t, \tau, x_{n}(\tau)\right)-\nu\left(t, \tau, x_{0}(\tau)\right) \Delta \tau\right)^{p} \Delta t\right)^{\frac{1}{p}} \rightarrow 0 .
$$

Therefor we have the compactness of our mapping. 
Lemma 5.7. Mapping $V$ given by (1) is continuously Gâteaux differentiable and a Gâteaux derivative $V^{\prime}(x): W_{\Delta, 0}^{1, p}\left([0,1]_{\mathbb{T}}, \mathbb{R}^{N}\right) \rightarrow L^{p}\left([0,1]_{\mathbb{T}}, \mathbb{R}^{N}\right)$ at any point $x \in W_{\Delta, 0}^{1, p}\left([0,1]_{\mathbb{T}}, \mathbb{R}^{N}\right)$ is given by

$$
V^{\prime}(x(t)) h(t):=h^{\Delta}(t)+\int_{0}^{t} \nu_{x}(t, \tau, x(\tau)) h(\tau) \Delta \tau,
$$

for every $h \in W_{\Delta, 0}^{1, p}\left([0,1]_{\mathbb{T}}, \mathbb{R}^{N}\right)$. Moreover, for any fixed $x \in W_{\Delta, 0}^{1, p}\left([0,1]_{\mathbb{T}}, \mathbb{R}^{N}\right)$ operator $V^{\prime}(x)$ is one to one and onto.

Proof. The formula for a derivative follows from (5.3) and the Lebesgue Dominated Convergence Theorem.

Let $\left(x_{n}\right)_{n \in \mathbb{N}}$ be a sequence from $W_{\Delta, 0}^{1, p}\left([0,1]_{\mathbb{T}}, \mathbb{R}^{N}\right)$ convergent to $x$. For every $h \in W_{\Delta, 0}^{1, p}\left([0,1]_{\mathbb{T}}, \mathbb{R}^{N}\right)$, by the Dominated Convergence Theorem we obtain

$$
\begin{aligned}
\left\|V^{\prime}(x) h-V^{\prime}\left(x_{n}\right) h\right\|_{L_{\Delta}^{p}} & =\left(\int_{0}^{1}\left|\int_{0}^{t}\left(\nu_{x}(t, \tau, x(\tau))-\nu_{x}\left(t, \tau, x_{n}(\tau)\right)\right) h(\tau) \Delta \tau\right|^{p} \Delta t\right)^{\frac{1}{p}} \\
& \leq\|h\|_{L_{\Delta}}\left(\int_{0}^{1} \int_{0}^{t}\left|\nu_{x}(t, \tau, x(\tau))-\nu_{x}\left(t, \tau, x_{n}(\tau)\right)\right|^{p} \Delta \tau \Delta t\right)^{\frac{1}{p}} \rightarrow 0 .
\end{aligned}
$$

Therefore $V^{\prime}(x)$ is also continuous.

In order to prove that for any fixed $x \in W_{\Delta, 0}^{1, p}\left([0,1]_{\mathbb{T}}, \mathbb{R}^{N}\right)$ operator $V^{\prime}(x)$ is one to one and onto we will show that for any fixed $\xi \in L_{\Delta}^{p}\left([0,1]_{\mathbb{T}}, \mathbb{R}^{N}\right)$ the following linear integro-differential equation

$$
h^{\Delta}(t)+\int_{0}^{t} \nu_{x}(t, \tau, x(\tau)) h(\tau) \Delta \tau=\xi(t) \text { for } \Delta \text {-a.e. } t \in[0,1]_{\mathbb{T}}
$$

has a unique solution in $W_{\Delta, 0}^{1, p}\left([0,1]_{\mathbb{T}}, \mathbb{R}^{N}\right)$. In order to do this let us fix $\xi \in L_{\Delta}^{p}\left([0,1]_{\mathbb{T}}, \mathbb{R}^{N}\right)$ and consider the following linear equation

$$
h^{\Delta}(t)=u(t)+\xi(t) \text { for } \Delta \text {-a.e. } t \in[0,1]_{\mathbb{T}},
$$

where $u \in L_{\Delta}^{p}\left([0,1]_{\mathbb{T}}, \mathbb{R}^{N}\right)$ is fixed for the time being. Such an equation has a unique solution as well, which will be denoted $h_{u} \in W_{\Delta, 0}^{1, p}\left([0,1]_{\mathbb{T}}, \mathbb{R}^{N}\right)$ and wich is given by

$$
h_{u}(t)=\int_{0}^{t} \xi(s)-u(s) \Delta s \text { for } \Delta \text {-a.e. } t \in[0,1]_{\mathbb{T}} .
$$

Now, consider the mapping $\Gamma: L_{\Delta}^{p}\left([0,1]_{\mathbb{T}}, \mathbb{R}^{N}\right) \rightarrow L_{\Delta}^{p}\left([0,1]_{\mathbb{T}}, \mathbb{R}^{N}\right)$ given by

$$
\Gamma: L_{\Delta}^{p}\left([0,1]_{\mathbb{T}}, \mathbb{R}^{N}\right) \ni u(\cdot) \mapsto \int_{0} \nu_{x}(\cdot, \tau, x(\tau)) h_{u}(\tau) \Delta \tau \in L_{\Delta}^{p}\left([0,1]_{\mathbb{T}}, \mathbb{R}^{N}\right),
$$


and the associated $u(\cdot)=(\Gamma u)(\cdot)$. We will show that $\Gamma$ is a contraction and thus it has an unique fixed point. For any $u_{1}, u_{2} \in L_{\Delta}^{p}\left([0,1]_{\mathbb{T}}, \mathbb{R}^{N}\right)$ we have

$$
\begin{aligned}
\left\|\Gamma u_{1}-\Gamma u_{2}\right\|_{\beta, L_{\Delta}^{p}}^{p}= & \int_{0}^{1} \frac{1}{e_{\beta}^{\sigma}(t, 0)}\left|\int_{0}^{t} \nu_{x}(t, \tau, x(\tau))\left(\int_{0}^{\tau} u_{1}(s)-u_{2}(s) \Delta s\right) \Delta \tau\right|^{p} \Delta t \\
\leq & \int_{0}^{1} \frac{1}{e_{\beta}^{\sigma}(t, 0)}\left(\int_{0}^{t}\left|\nu_{x}(t, \tau, x(\tau))\right|^{q} \Delta \tau\right)^{\frac{p}{q}} \times \\
& \times\left(\int_{0}^{t} \int_{0}^{\tau}\left|u_{1}(s)-u_{2}(s)\right|^{p} \Delta s \Delta \tau\right) \Delta t \\
& \leq H^{\frac{p}{q}} \frac{1}{\beta^{2}}\left\|u_{1}-u_{2}\right\|_{\beta, L_{\Delta}^{p}}^{p},
\end{aligned}
$$

where $H \geq\|x\|_{L_{\Delta}^{\infty}}^{q} \int_{0}^{1} c_{2}^{q}(t, \tau) \Delta \tau$. Hence

$$
\left\|\Gamma u_{1}-\Gamma u_{2}\right\|_{\beta, L_{\Delta}^{p}} \leq \frac{H^{\frac{1}{q}}}{\beta^{\frac{2}{p}}}\left\|u_{1}-u_{2}\right\|_{\beta, L_{\Delta}^{p}} .
$$

For $\beta \geq H^{\frac{p}{2 q}}$ the mapping $\Gamma$ is a contraction so by the Banach Principle it has fixed point $u^{*} \in L_{\Delta}^{p}\left([0,1]_{\mathbb{T}}, \mathbb{R}^{N}\right)$ Therefore for every $\xi \in L_{\Delta}^{p}\left([0,1]_{\mathbb{T}}, \mathbb{R}^{N}\right)$ there exist $u^{*} \in L_{\Delta}^{p}\left([0,1]_{\mathbb{T}}, \mathbb{R}^{N}\right)$ which is a solution to $u=\Gamma u$. Hence, there is unique $h \in W_{\Delta, 0}^{1, p}\left([0,1]_{\mathbb{T}}, \mathbb{R}^{N}\right)$ satisfying

$$
h^{\Delta}(t)=\xi(t)-u^{*}(t) \Delta \text {-a.e. on }[0,1]_{\mathbb{T}},
$$

which depends solely on the choice of $\xi \in L_{\Delta}^{p}\left([0,1]_{\mathbb{T}}, \mathbb{R}^{N}\right)$.

Lemma 5.8. Operator $V: W_{\Delta, 0}^{1, p}\left([0,1]_{\mathbb{T}}, \mathbb{R}^{N}\right) \rightarrow L^{p}\left([0,1]_{\mathbb{T}}, \mathbb{R}^{N}\right)$ satisfies the norm coercivity condition.

Proof. We see that

$$
\begin{aligned}
\left\|\int_{0} \nu(\cdot, \tau, x(\tau)) \Delta \tau\right\|_{\beta, L_{\Delta}^{p}} & \leq\left(\int_{0}^{1} \frac{1}{e_{\beta}^{\sigma}(t, 0)}\left|\int_{0}^{t} c_{1}(t, \tau)\right| x(\tau)\left|+d_{1}(t, \tau) \Delta \tau\right|^{p} \Delta t\right)^{\frac{1}{p}} \\
& \leq H_{1}^{\frac{1}{q}}\left(\int_{0}^{1} \frac{1}{e_{\beta}^{\sigma}(t, 0)} \int_{0}^{t}|x(\tau)|^{p} \Delta \tau \Delta t\right)^{\frac{1}{p}}+\left\|d_{1}\right\|_{\beta, L_{\Delta}^{p}} \\
& \leq H_{1}^{\frac{1}{q}}\left(\frac{1}{\beta}\right)^{\frac{2}{p}}\|x\|_{\beta, W_{\Delta, 0}^{1, p}}+\left\|d_{1}\right\|_{\beta, L_{\Delta}^{p}} .
\end{aligned}
$$


As a consequence

$$
\begin{aligned}
\|V(x)\|_{\beta, L_{\Delta}^{p}} & \geq\|x\|_{\beta, W_{\Delta, 0}^{1, p}}-\left\|\int_{0}^{\cdot} \nu(\cdot, \tau, x(\tau)) \Delta \tau\right\|_{\beta, L_{\Delta}^{p}} \\
& \geq\left(1-H_{1}^{\frac{1}{q}}\left(\frac{1}{\beta}\right)^{\frac{2}{p}}\right)\|x\|_{\beta, W_{\Delta, 0}^{1, p}}-\left\|d_{1}\right\|_{\beta, L_{\Delta}^{p}} .
\end{aligned}
$$

Thus choosing $\beta>\max \left\{H_{1}^{\frac{p}{2 q}}, H^{\frac{p}{2 q}}\right\}$, we se that the assertion holds.

Theorem 5.9. Operator $V$ defined by (2) is a diffeomorphism.

Proof. We show that for any $x \in W_{\Delta, 0}^{1, p}\left([0,1]_{\mathbb{T}}, \mathbb{R}^{N}\right)$ there exists $C_{x}>0$ such that

$$
\left\|V^{\prime}(x) h\right\| \geq C_{x}\|h\|
$$

for any $h \in W_{\Delta, 0}^{1, p}\left([0,1]_{\mathbb{T}}, \mathbb{R}^{N}\right)$. Let us choose $H$ and $\beta$ as in the proof of Lemma 5.7. Then

$$
\left\|V^{\prime}(x) h\right\|_{\beta, L_{\Delta}^{p}} \geq\left(1-H^{\frac{1}{q}}\left(\frac{1}{\beta}\right)^{\frac{2}{p}}\right)\|h\|_{\beta, W_{\Delta, 0}^{1, p}}
$$

and we can define a positive $C_{x}:=1-H^{\frac{1}{q}}\left(\frac{1}{\beta}\right)^{\frac{2}{p}}$. Additionally, we showed in the proof of Lemma 5.7 that Fréchet derivative of function $V$ is one to one and onto. Therefor $V$ is a local diffeomorphism.

Operator $V$ as the sum of the proper operator and the compact operator is proper. Recalling that $V$ is norm coercive we see that operator $V$ is diffeomorphism.

Theorem 5.10. Assume that conditions (B1)-(B4) are satisfied. For any fixed $y \in L_{\Delta}^{p}\left([0,1]_{\mathbb{T}}, \mathbb{R}^{N}\right)$ problem $(2)$ has exactly one solution.

Proof. The assertion from Theorem 5.9.

Acknowledgment. The current form of the article is simplified by the observation made by Prof. W. Kryszewski concerning the usage of the main tool from [13].

\section{References}

[1] R. A. Adams and J. J. F. Fournier. Sobolev spaces, volume 140 of Pure and Applied Mathematics (Amsterdam). Elsevier/Academic Press, Amsterdam, 2003. 
[2] R. P. Agarwal, V. Otero-Espinar, K. Perera, and D. R. Vivero. Basic properties of Sobolev's spaces on time scales. Adv. Difference Equ. pages Art. ID 38121, 14, 2006.

[3] L. Berezansky, M. Migda, and E. Schmeidel. Some stability conditions for scalar Volterra difference equations. Opuscula Math., 36(4):459-470, 2016.

[4] M. Bohner and A. Peterson. Dynamic equations on time scales. Birkhäuser Boston, Inc., Boston, MA, 2001.

[5] M. Bohner and A. Peterson. Advances in dynamic equations on time scales. Birkhäuser Boston, Inc., Boston, MA, 2003

[6] I. L. D. dos Santos. On Volterra integral equations on time scales. Mediterr. J. Math., 12(2):471-480, 2015.

[7] P. Drábek and J. Milota. Methods of nonlinear analysis. Birkhäuser/Springer Basel AG, Basel, 2013.

[8] M. Galewski and M. Koniorczyk. On a global implicit function theorem and some applications to integro-differential initial value problems. Acta Math. Hungar., 148(2):257-278, 2016.

[9] S. G. Georgiev. Integral equations on time scales. volume 5 of Atlantis Studies in Dynamical Systems. Atlantis Press, [Paris], 2016.

[10] E. Ghahraei, S. Hosseini, and M. R. Pouryayevali. Pseudo-Jacobian and global inversion of nonsmooth mappings on Riemannian manifolds. Nonlinear Anal., 130:229-240, 2016.

[11] S. Hamadène. Viscosity solutions of second order integral-partial differential equations without monotonicity condition: a new result. Nonlinear Anal., 147:213-235, 2016.

[12] S. Hilger. Ein maßkettenkalkül mit anwendung auf zentrumsmannigfaltigkeiten. 1989.

[13] D. Idczak, A. Skowron, and S. Walczak. On the diffeomorphisms between Banach and Hilbert spaces. Adv. Nonlinear Stud., 12(1):89-5100, 2012.

[14] D. B. Pachpatte. Fredholm type integrodifferential equation on time scales. Electron. J. Differential Equations, pages 10, No. 140, 2010.

[15] D. B. Pachpatte. Integral inequalitys for partial dynamic equations on time scales. Differential Equations, pages 7, No. 50, 2012. 
[16] B. P. Rynne. $L^{2}$ spaces and boundary value problems on time-scales. Math. Anal. Appl., 328(2):1217-1236, 2007.

[17] K. Yosida. Functional analysis. Springer-Verlag, Berlin-New York, 1978. Grundlehren der Mathematischen Wissenschaften, Band 123.

[18] E. Zeidler. Nonlinear functional analysis and its applications. I. SpringerVerlag, New York, 1986.

Bartłomiej Kluczyński

Institute of Mathematics,

Lodz University of Technology,

Wolczanska 215, 90-924 Lodz, Poland.

Email: Bartlomiej.G.Kluczynski@gmail.com 